\title{
Heterogeneous Basket Options Pricing Using Analytical Approximations
}

\author{
Georges Dionne \\ HEC Montréal, Canada \\ Geneviève Gauthier \\ HEC Montréal, Canada \\ Nadia Ouertani \\ LEFA, ISCAE Manouba University, Tunisia \\ Nabil Tahani \\ York University, Canada
}

This paper proposes the use of analytical approximations to price an heterogeneous basket option combining commodity prices, foreign currencies and zero-coupon bonds. The performance of three moment matching approximations is examined: inverse gamma, Edgeworth expansion around the lognormal and Johnson family distributions. Since there is no closed-form formula for basket options, Monte Carlo simulations are carried out to generate the benchmark values. A simulation experiment on a set of options based on a random choice of parameters is performed. The results show that the Edgeworth-lognormal and Johnson distributions give the most accurate results. (JEL: C15, C63, F31, G13).

* The authors acknowledge the financial support of the Institut de Finance Mathématiques de Montréal, (IFM2), the National Science and Engineering Research Council of Canada (NSERC) and the Fonds Québécois de Recherche sur la Société et la Culture, (FQRSC). This paper extends N. Ouertani's Ph.D. thesis on which Phelim P. Boyle, Michel Denault, and Pascal François have made very helpful suggestions. The authors thank Moshe A. Milevsky and Chris Robinson for their comments, and are also grateful for the feedback from an anonymous referee and the editor-in-chief Panayiotis Theodossiou. Previous versions of this paper have been presented at the 18th Annual Australasian Finance and Banking Conference (Sydney, December 2005), the Northern Finance Association Meeting (Vancouver, October 2005), the Bachelier Finance Society, 4th World congress, (Tokyo, August 2006), the European Financial Management Association Annual Meeting, (Madrid, June 2006) and the Conference of the Swiss Society for Financial Market Research, (Zurich, April 2006)

(Multinational Finance Journal, 2011, vol. 15, no. 1/2, pp. 47-85)

(C) Multinational Finance Society, a nonprofit corporation. All rights reserved.

DOI: $10.17578 / 15-1 / 2-2$ 
Keywords: Basket Options, Options Pricing, Analytical Approximations, Monte Carlo Simulation

\section{Introduction}

The use of derivative securities in risk management activities emerged in the early 1990s and has evolved rapidly since. They now are the most important tool in financial risk management. According to the 1998 Wharton school survey of financial risk management by US non-financial firms, over 50\% of the responding firms use derivatives products to hedge their exposure, $83 \%$ of them use derivatives to hedge foreign-exchange risk, $76 \%$ to hedge interest-rate risk, and $56 \%$ to hedge commodity-price risk. ${ }^{1}$ In the current economic situation, many non-financial institutions such as gold-mining firms, energy companies or airlines companies may face different financial risks simultaneously and hence look for the most efficient way to hedge their portfolios.

For example, gold-mining firms may be exposed to different types of risk: commodity risk, which can include uncertainty about the price of their primary product, gold, as well as their by-products such as silver and copper; since these firms sell their products in other countries, they are exposed to currency risk; and their interest rate risk exposure is primarily related to their fixed-rate and variable-rate debt. Though all these markets are very active and very liquid so that a firm could hedge all its different risk exposures separately, it would be more interesting to adopt a portfolio approach because it allows the firm to account for the correlations between these different financial markets and hedge simultaneously a great variety of different financial risks. To attain this goal, basket options are an efficient instrument to use.

Basket options are a type of exotic option whose payoff depends on the value of a basket of assets. They offer the flexibility of being able to include virtually any kind and any number of assets, and hence can suitably respond to the specific needs of a firm in hedging its risk exposures. A risk manager may have other incentives in using basket options: depending on the design of each option, they are usually cheaper than a portfolio of standard options. In practice, basket options are traded over-the-counter and are designed specifically to meet the needs of the buyer. For these reasons, the liquidity premium required by the counterpart may annihilate some of the advantages coming from the correlation structure of the basket.

The pricing of basket options is more challenging than that of

1. Financial Management, Vol. 27, No. 4, winter 1998. 
standard options because there is no explicit analytical solution for the density function of a weighted sum of correlated assets. Several approaches are proposed in the literature to price basket options. They can be categorized as follows:

Numerical methods: Monte Carlo simulations [Barraquand (1995), Pellizzari (2001)], Quasi-Monte Carlo [Dahl (2000) and Dahl and Benth (2001)], and multinomial trees [Rubinstein (1994), Wan(2002)],

Upper and lower bounds: Curran (1994), Vanmaele, Deelstra and Liinev (2004), Laurence and Wang (2004) and Deelstra, Liinev and Vanmaele (2004),

Analytical approximations: Gentle (1993), Huynh (1994), Milevsky and Posner (1998a, 1998b, 1999), Posner and Milevsky (1999), Ju (2002), Datey, Gauthier and Simonato (2003) and Brigo et al. (2004).

However, all these papers are based on two main hypotheses. They assume constant interest rates and homogeneous basket options; that is, all the assets underlying the basket are of the same nature and thus hedge the same type of risk. Unfortunately, this homogeneity in basket assets does not always match a firm's needs, and with a growing diversification in investors' portfolios, heterogeneous basket options become a very efficient tool to hedge multiple risk exposures simultaneously.

This article proposes analytical approximations to price heterogeneous basket options, consisting of commodities, foreign currencies and zero-coupon bonds, with stochastic interest rates, and compares the accuracy and the performance of these approximations for different sets of parameters. Three distributions based on the moment matching technique will be used to approximate the basket density function: inverse gamma distribution, Edgeworth expansion around the lognormal distribution and Johnson distribution. It is found that the Edgeworth-lognormal and Johnson approximations perform better than the inverse gamma approximation. The contributions of this article are: (1) to design an arbitrage-free framework in which the domestic and the foreign interest rates, the exchange rate, the commodity price and the convenience yield are stochastic, (2) to compute the moments of the heterogeneous basket under the $T$-forward measure, (3) to show that some of the existing analytical approximations may be used in this very general setting and (4) to quantify the approximation errors.

The next section presents the pricing model under the forward measure. Section III derives the inverse gamma, the Edgeworth-lognormal and the Johnson approximations. Section IV compares the performance of the three approximations. Section V discusses some extensions and Section VI concludes. 


\section{Pricing of a European Heterogeneous Basket Option}

Let us consider a non-financial firm looking for an alternative way to simultaneously hedge its different financial risk exposures: commodity price risk, exchange rate risk and interest rate risk. In the following, $S_{t}$ denotes the value of the commodity at time $t, \delta_{t}$ is its continuously-compounded convenience yield at time $t, C_{t}$ is the value at time $t$ of one unit of the foreign currency expressed in the domestic currency, $P\left(t, T_{1}\right)$ is the time- $t$ value of a zero-coupon bond paying one unit of the domestic currency at time $T_{1}, P^{*}\left(t, T_{2}\right)$ is the time- $t$ value of a foreign zero-coupon bond paying one unit of the foreign currency at time $T_{2}$. The firm's financial assets dynamics under the historical probability measure $P$ are given by the following stochastic differential equations $^{2}$ (SDE hereafter):

$$
\begin{gathered}
d S_{t}=S_{t}\left[\left(\alpha_{s}-\delta_{t}\right) d t+\sigma_{s} d W_{t}^{(1)}\right] \\
d \delta_{t}=\kappa\left(\theta-\delta_{t}\right) d t+\sigma_{\delta} d W_{t}^{(1)} \\
d C_{t}=C_{t}\left[\alpha_{c} d t+\sigma_{c} d W_{t}^{(2)}\right] \\
d P\left(t, T_{1}\right)=P\left(t, T_{1}\right)\left[\left(r_{t}-\beta_{t, T_{1}}\left(\frac{\gamma_{t, T_{1}}}{\eta_{t, T_{1}}}-\beta_{t, T_{1}}\right)\right) d t-\beta_{t, T_{1}} d W_{t}^{(3)}\right] \\
0 \leq t \leq T_{1}, \\
d P^{*}\left(t, T_{2}\right)=P^{*}\left(t, T_{2}\right)\left[\left(r_{t}^{*}-\beta_{t, T_{2}}^{*}\left(\frac{\gamma_{t, T_{2}}^{*}}{\eta_{t, T_{2}}^{*}}-\beta_{t, T_{2}}^{*}\right)\right) d t-\beta_{t, T_{2}}^{*} d W_{t}^{(4)}\right] \\
0 \leq t \leq T_{2} .
\end{gathered}
$$

2. In this setting, the convenience yield and the commodity price share the same source of risk to ensure the market completeness. For more details, see Dionne, Gauthier and Ouertani (2008). 
The expressions for the bond prices are obtained from the domestic and the foreign instantaneous forward rates:

$$
\begin{gathered}
d f\left(t, T_{1}\right)=\gamma_{t, T_{1}} d t+\eta_{t, T_{1}} d W_{t}^{(3)}, 0 \leq t \leq T_{1}, \\
d f^{*}\left(t, T_{2}\right)=\gamma_{t, T_{2}}^{*} d t+\eta_{t, T_{2}}^{*} d W_{t}^{(4)}, 0 \leq t \leq T_{2},
\end{gathered}
$$

in which the volatility parameters $\eta_{t, T_{1}}$ and $\eta_{t, T_{2}}^{*}$ are deterministic functions of time $t$ and maturity. Consequently, $\beta_{t, T_{1}}=\int_{t}^{T_{1}} \eta_{t, s} d s, \beta_{t, T_{2}}^{*}=$ $\int_{t}^{T_{2}} \eta_{t, s}^{*} d s$ and $r_{t}=f(t, t), r_{t}^{*}=f^{*}(t, t)$ are respectively the continuouslycompounded domestic and foreign risk-free spot interest rates. The drift terms $\gamma_{t, T_{1}}$ and $\gamma_{t, T_{2}}^{*}$ are not specified since they do not appear in the pricing formulae. The four-dimensional Brownian motion $W=\left(W^{(1)}, W^{(2)}, W^{(3)}, W^{(4)}\right)^{\prime}$ is constructed on a filtered probability space $\left(\Omega, \mathcal{F},\left\{\mathcal{F}_{t}: t \geq 0\right\}, P\right)$ with the following correlation structure:

$$
\operatorname{Corr}^{P}\left(W_{t}^{(i)}, W_{t}^{(j)}\right)=\rho_{i, j}, \text { for each } i, j=\{1,2,3,4\} \text { and } t>0
$$

Consider a European call option that gives the firm the right to buy a basket consisting of the commodity, the domestic zero-coupon bond and the foreign zero-coupon bond expressed in domestic currency units, at a strike price $K_{B}$. Its payoff at maturity $T$ is given by:

$$
X_{B}=\max \left[B_{T}-K_{B}, 0\right],
$$

where the basket value at time $t$ is $B_{t}=w_{1} S_{t}+w_{2} P\left(t, T_{1}\right)+$ $w_{3} C_{t} P^{*}\left(t, T_{2}\right), 0 \leq t \leq T \leq T_{1}, T_{2}, w_{1}, w_{2}$ and $w_{3}$ correspond to the numbers of shares invested respectively in the commodity $S_{T}$, the domestic zero-coupon bond $P\left(T, T_{1}\right)$ and the foreign zero-coupon bond expressed in domestic currency units $C_{T} P^{*}\left(T, T_{2}\right)$. Since the proposed market model is complete, ${ }^{3}$ Harrison and Pliska (1981) allows the pricing of any contingent claim as the expectation of the discounted payoff under the risk-neutral measure $Q$. Consequently, the price of the

3. The derivation of the unique risk-neutral measure is available upon request. 
European call option at time $t$ is given by:

$$
\begin{aligned}
V_{t}^{B}= & E^{Q}\left[\exp \left(-\int_{t}^{T} r_{u} d u\right) \max \left(B_{T}-K_{B}, 0\right) \mid \mathcal{F}_{t}\right] \\
& =P(t, T) E^{Q_{T}}\left[\max \left(B_{T}-K_{B}, 0\right) \mid \mathcal{F}_{t}\right] \\
& =P(t, T) \int_{-\infty}^{+\infty} \max \left(x-K_{B}, 0\right) v(x) d x
\end{aligned}
$$

where $v(x)$ is the true (unknown) density function of the basket value $B_{T}$ under the $T$-forward measure $Q_{T}$. This forward measure has a Radon-Nikodym derivative with respect to $Q$ denoted by $\frac{d Q_{T}}{d Q}$, and its associated $Q$ - martingale is given by:

$$
\zeta_{t}=\mathrm{E}^{Q}\left(\frac{d Q_{T}}{d Q} \mid \mathcal{F}_{t}\right)=\frac{\exp \left(-\int_{t}^{T} r_{u} d u\right)}{P(t, T)} .
$$

The SDEs satisfied by the basket underlying assets under the $T$-forward measure $Q_{T}$ is given in appendix A, and its strong solution is obtained for any $0 \leq t \leq T$ as:

$$
\begin{gathered}
S_{T}=S_{t} \exp \left(\int_{t}^{T}\left(r_{u}-\delta_{u}-\frac{\sigma_{s}^{2}}{2}-\sigma_{s} \rho_{1,3} \beta_{u, T}\right) d u+\sigma_{s} \int_{t}^{T} d \hat{W}_{u}^{(1)}\right), \quad(3 \mathrm{a}) \\
\delta_{T}=\left(\begin{array}{c}
\left.\delta_{t} e^{-\kappa(T-t)}+\left(\kappa \theta-\frac{\sigma_{\delta}}{\sigma_{S}} \alpha_{S}\right) \frac{1-e^{-\kappa(T-t)}}{\kappa}-\sigma_{\delta} \rho_{1,3} \int_{t}^{T} e^{-\kappa(T-u)} \beta_{u, T} d u\right), \\
+\frac{\sigma_{\delta}}{\sigma_{s}} \int_{t}^{T} r_{u} e^{-\kappa(T-u)} d u+\sigma_{\delta} \int_{t}^{T} e^{-\kappa(T-u)} d \hat{W}_{u}^{(1)}
\end{array}\right),
\end{gathered}
$$




$$
\begin{gathered}
C_{T}=C_{t} \exp \left(\int_{t}^{T}\left(r_{u}-r_{u}^{*}-\frac{\sigma_{c}^{2}}{2}-\sigma_{c} \rho_{2,3} \beta_{u, T}\right) d u+\sigma_{c} \int_{t}^{T} d \hat{W}_{u}^{(2)}\right), \quad(3 \mathrm{c}) \\
P\left(T, T_{1}\right)=P\left(t, T_{1}\right) \exp \left(\int_{t}^{T}\left(r_{u}-\frac{1}{2} \beta_{u, T_{1}}^{2}+\beta_{u, T_{1}} \beta_{u, T}\right) d u-\int_{t}^{T} \beta_{u, T_{1}} d \hat{W}_{u}^{(3)}\right),
\end{gathered}
$$

$P^{*}\left(T, T_{2}\right)=$

$$
P^{*}\left(t, T_{2}\right) \exp \left(\begin{array}{l}
\int_{t}^{T}\left(r_{u}^{*}-\frac{1}{2}\left(\beta_{u, T_{2}}^{*}\right)^{2}+\sigma_{c} \rho_{2,4} \beta_{u, T_{2}}^{*}+\rho_{3,4} \beta_{u, T_{2}}^{*} \beta_{u, T}\right) d u \\
-\int_{t}^{T} \beta_{u, T_{2}}^{*} d \hat{W}_{u}^{(4)}
\end{array}\right),(3 \mathrm{e})
$$

where $\hat{W}=\left(\hat{W}^{(1)}, \hat{W}^{(2)}, \hat{W}^{(3)}, \hat{W}^{(4)}\right)^{\prime}$ is a four-dimensional $Q_{T}-$ Brownian motion with the same correlation structure $\operatorname{Corr}^{Q_{T}}\left(\hat{W}_{t}^{(i)}, \hat{W}_{t}^{j}\right)=\rho_{i, j}$.

The evaluation of the basket call option is complicated by the absence of a closed-form equation for the density function $v(x)$ in equation (2). Among the different approaches proposed in the basket options literature, we find some numerical techniques such as Monte Carlo, Quasi-Monte Carlo and lattice-based method, the upper and lower bound computations, and some analytical approximations. ${ }^{4}$

Lattice-based approaches are widely used for options on a single asset. They are exponentially complicated and computationally expensive for options on multiple assets. For example, a three-asset basket option needs $(n+1)^{3}$ terminal nodes on an $n$-step trinomial tree. On the other hand, Monte Carlo and Quasi-Monte Carlo methods can be used for multi-assets options and are less time-consuming than latticebased approaches. The estimates can be as accurate as needed at a computational cost however; to improve the accuracy of an $n$-path simulation by one half, one needs to simulate $4 n$ paths and thus needs 4 times more computing time.

A practitioner might be interested in slightly less accurate but very

4. The upper and lower bounds methods are not useful in this case since the market model proposed is complete and thus a unique option price can be computed. 
fast methods such as analytical approximations. These methods approximate the unknown basket density function with an alternative and easy-to-compute distribution. In the next sections, three well-known analytical approximations will be extended to heterogeneous basket options: the inverse gamma distribution, the Edgeworth expansion around the lognormal distribution and Johnson distribution.

\section{Analytical Approximations}

In order to apply these moment matching-based approximations, we need to calculate the first four moments of the weighted sum underlying the European option under the $T$-forward measure $Q_{T}$. The following notations are adopted:

$$
\begin{gathered}
\mu_{n}^{\prime}(h)=\int_{-\infty}^{+\infty} x^{n} h(x) d x, \\
\mu_{n}(h)=\int_{-\infty}^{+\infty}\left(x-\mu_{1}^{\prime}(h)\right)^{n} h(x) d x,
\end{gathered}
$$

where $\mu_{n}^{\prime}(h)$ and $\mu_{n}(h)$ represent respectively the $n^{\text {th }}$ non-centered and centered moments of a density function $h \in\{v, a\}$. The case $h=v$ corresponds to the exact density of the basket value under the forward measure, while $h=a$ corresponds to the approximate density. The first four cumulants of the distribution $h$, i.e. the mean, the variance, the skewness and kurtosis are defined as:

$$
\begin{gathered}
\kappa_{1}(h)=\mu_{1}^{\prime}(h), \\
\kappa_{2}(h)=\mu_{2}(h), \\
\kappa_{3}(h)=\mu_{3}(h), \\
\kappa_{4}(h)=\mu_{4}(h)-3 \mu_{2}(h) .
\end{gathered}
$$


Lemma 1: For any positive integer $n$, the $n^{\text {th }}$ non-centered moment of the true distribution of the weighted sum $B_{T}$ under the $T$-forward measure $Q_{T}$ is

$$
\begin{aligned}
\mu_{n}^{\prime}(v)= & E^{Q_{T}}\left[B_{T}^{n}\right]=E^{Q_{T}}\left[\left(w_{1} S_{T}+w_{2} P\left(T, T_{1}\right)+w_{3} C_{T} P^{*}\left(T, T_{2}\right)\right)^{n}\right] \\
= & \sum_{k=0}^{n} \sum_{j=0}^{k} \frac{n !}{j !(k-j) !(n-k) !} w_{1}^{j} w_{2}^{(k-j)} w_{3}^{(n-k)} E^{Q_{T}}\left[S_{T}^{j}\left(P\left(T, T_{1}\right)\right)^{(k-j)}\right. \\
& \left.C_{T}^{n-k}\left(P^{*}\left(T, T_{2}\right)\right)^{n-k}\right] .
\end{aligned}
$$

Note that the lognormal distribution of $S_{T}, P\left(T, T_{1}\right), C_{T}$, and $P^{*}\left(T, T_{2}\right)$ under the forward measure allows the computation of the last expectation in lemma 1 by using the following identity:

$$
E[\exp (\mu+\sigma Z)]=\exp \left(\mu+\frac{\sigma^{2}}{2}\right) \text { where } Z \sim N(0,1) \text {. }
$$

Details are given in appendix B.

\section{A. Inverse Gamma Approximation}

In this section, the inverse gamma distribution is used to approximate the sum of correlated lognormal variables. The approximation was first used by Milevsky and Posner (1998a, 1998b) to price Asian and basket options. In fact, a sum of correlated lognormal variables converges asymptotically to an inverse gamma variable. Under an inverse gamma distribution for the underlying basket, a European basket call option has a closed-form solution that looks like a Black and Scholes (1973) (B\&S hereafter) formula:

$$
V_{\text {gamma }}^{B}=P(t, T)\left(\mu_{1}^{\prime}(v) G\left(\frac{1}{K_{B}} \mid \alpha-1, \beta\right)-K_{B} G\left(\frac{1}{K_{B}} \mid \alpha, \beta\right)\right),
$$

where $G(\bullet \mid \alpha, \beta)$ is the cumulative function of a gamma distribution with parameters $(\alpha, \beta)$. These parameters are determined by matching 
the first two moments of the exact and the approximate distributions to obtain:

$$
\alpha=\frac{\mu_{1}^{\prime 2}(v)-2 \mu_{2}^{\prime}(v)}{\mu_{1}^{\prime 2}(v)-\mu_{2}^{\prime}(v)} \text { and } \beta=\frac{\mu_{2}^{\prime}(v)-\mu_{1}^{\prime 2}(v)}{\mu_{1}^{\prime}(v) \mu_{2}^{\prime}(v)} \text {. }
$$

Mathematical details of the pricing formula are provided in appendix $\mathrm{C}$.

\section{B. Edgeworth Expansion Around the Lognormal Distribution}

We now present an analytical approximation based on a generalized Edgeworth expansion around the lognormal distribution. This approach, introduced by Jarrow and Rudd (1982) in option pricing, substitutes an unknown density function $v(\bullet)$ with a Taylor-like expansion around an easy-to-use density function denoted by $a(\bullet)$. Note, however, that Edgeworth expansions usually lead to a function which is not a true density function. Barton and Denis (1952) derive some conditions on the third and fourth moments of the unknown distribution to guarantee that the approximation obtained with a truncated Edgeworth expansion is positive and unimodal.

Moreover, Ju (2002) points out that the Edgeworth expansion may diverge for some parameter values, which consequently can give incorrect prices for high volatility and long maturity options. However, in this article the use of the Edgeworth expansion did not lead to this problem.

Following Huynh (1994) who uses this approach for basket options, an Edgeworth expansion of order 4 is used and the first two moments of the exact and the lognormal distributions are matched. Under this approximation, a European basket call option can be obtained as a Black and Scholes price adjusted for the excess skewness and the excess kurtosis from the lognormal density:

$$
\begin{aligned}
V_{\log \text { normal }}^{B} & =P(t, T)\left[V-\frac{\kappa_{3}(v)-\kappa_{3}(a)}{3 !} \frac{d a\left(K_{B}\right)}{d x}\right. \\
& \left.+\frac{\kappa_{4}(v)-\kappa_{4}(a)}{4 !} \frac{d^{2} a\left(K_{B}\right)}{d x^{2}}\right],
\end{aligned}
$$

where

$$
V=\mu^{\prime}(v) N\left(d_{1}\right)-K_{B} N\left(d_{2}\right),
$$




$$
\begin{gathered}
d_{1}=d_{2}+\beta, \\
d_{2}=\frac{\alpha-\ln \left(K_{B}\right)}{\beta}, \\
\alpha=\ln \left(\mu_{1}^{\prime 2}(v)\right)-\frac{1}{2} \ln \left(\mu_{1}^{\prime 2}(v)+\mu_{2}(v)\right), \\
\beta=\sqrt{\ln \left(1+\mu_{2}(v)\left(\mu_{1}^{\prime}(v)\right)^{-2}\right)},
\end{gathered}
$$

$a(\bullet)$ is the density function of a lognormal distribution (see equation (19) in appendix D), and $N(\bullet)$ represents the cumulative function of the standard normal distribution. The third and fourth moments of the lognormal distribution needed for the Edgeworth expansion depend only on the first and second moments of the exact distribution and are given by:

$$
\mu_{3}^{\prime}(a)=\left(\frac{\mu_{2}^{\prime}(v)}{\mu_{1}^{\prime}(v)}\right)^{3} \text { and } \mu_{4}^{\prime}(a)=\frac{\mu_{2}^{\prime}(v)^{6}}{\mu_{1}^{\prime}(v)^{8}}
$$

Details about the Edgeworth approximation formula are given in appendix D.

\section{Johnson Approximation}

Johnson (1949) proposes a family of density functions, obtained via a transformation of a standard normal variable that can be used to approximate unknown distributions. Let $Z$ be a standard normal variable and $X$ be a random variable with an unknown density function, Johnson (1949) suggests the following transformations between $Z$ and $X$ :

$$
\begin{aligned}
& Z=\gamma+\delta \psi\left(\frac{X-\varepsilon}{\lambda}\right) \\
& X=\varepsilon+\lambda \psi^{-1}\left(\frac{Z-\gamma}{\delta}\right)
\end{aligned}
$$


where $\gamma$ and $\delta$ are the shape parameters of the Johnson distribution, $\lambda$ is the scale parameter, $\mathcal{\varepsilon}$ is the threshold parameter, and $\psi(\bullet)$ is one of the following Johnson functions:

$$
\text { Lognormal system: } \psi_{L}(x)=\ln (x) \text {, }
$$

$$
\begin{gathered}
\text { Unbounded system: } \psi_{U}(x)=\ln \left(x+\sqrt{x^{2}+1}\right) \text {, } \\
\text { Bounded system: } \psi_{B}(x)=\ln \left(\frac{x}{1-x}\right) .
\end{gathered}
$$

The choice of the system and fitting parameters provides a great flexibility in adjusting the curve to match the first four moments of the unknown distribution. We use the lognormal $\left(\psi_{L}\right)$ and the unbounded $\left(\psi_{U}\right)$ systems that are common in the literature. The Hill, Hill and Holder (1976) algorithm, based on the true skewness and kurtosis of the basket distribution, is applied to determine which of the Johnson systems $\left(\psi_{L}\right.$ or $\left.\psi_{U}\right)$ should be used in the approximation. Unlike the approximations obtained with a truncated Edgeworth expansion, those based on Johnson (1949) systems correspond to true density functions with a perfect match of the first four moments.

Following Posner and Milevsky (1999), we substitute the unknown distribution of the underlying basket with the lognormal and the unbounded Johnson functions where the system parameters are calculated by matching the four moments. Under a Johnson density function, a European basket call option can be priced as:

$$
\begin{gathered}
V_{\text {Johnson }}^{B}=P(t, T) \int_{0}^{+\infty}\left(x-K_{B}\right) \psi(x) d x \\
=P(t, T)\left(\int_{0}^{+\infty} x \psi(x) d x-K_{B} \int_{0}^{+\infty} \psi(x) d x-\int_{0}^{K_{B}}\left(x-K_{B}\right) \psi(x) d x\right) \\
=P(t, T)\left(\mu_{1}^{\prime}(v)-K_{B}+\int_{0}^{K_{B}}\left(\int_{0}^{x} \psi(y) d y\right) d x\right)
\end{gathered}
$$




$$
\cong P(t, T)\left(\mu_{1}^{\prime}(v)-K_{B}+\int_{-\infty}^{K_{B}}\left(\int_{0}^{x} \psi(y) d y\right) d x\right) .
$$

The third line in the equation is obtained using an integration by parts. Milevsky and Posner (1999) show that the last double integral, involving a Johnson density function, can be computed as follows:

1. Lognormal system $\psi_{L}: X=\varepsilon+\lambda \exp \left(\frac{Z-\gamma}{\delta}\right)$

$$
\begin{gathered}
\int_{-\infty}^{K_{B}}\left(\int_{0}^{x} \psi(y) d y\right) d x=\left(K_{B}-\varepsilon\right) N\left(\gamma+\delta \ln \left(\frac{K_{B}-\varepsilon}{\lambda}\right)\right) \\
-\lambda \exp \left(\frac{1-2 \gamma \delta}{2 \delta^{2}}\right) N\left(\gamma+\delta \ln \left(\frac{K_{B}-\varepsilon}{\lambda}\right)-\frac{1}{\delta}\right) .
\end{gathered}
$$

2. Unbounded system $\psi_{U}: X=\varepsilon+\lambda \sinh \left(\frac{Z-\gamma}{\delta}\right)$

$$
\begin{aligned}
\int_{-\infty}^{K_{B}}\left(\int_{0}^{X} \psi(y) d y\right) d x & =\left(K_{B}-\varepsilon\right) N(q)+\frac{\lambda}{2} \exp \left(\frac{1}{2 \delta^{2}}\right) \exp \left(\frac{\gamma}{\delta}\right) N\left(q+\frac{1}{\delta}\right) \\
& -\frac{\lambda}{2} \exp \left(\frac{1}{2 \delta^{2}}\right) \exp \left(-\frac{\gamma}{\delta}\right) N\left(q-\frac{1}{\delta}\right)
\end{aligned}
$$

where $q=\gamma+\delta \sinh ^{-1}\left(\frac{K_{B}-\varepsilon}{\lambda}\right){ }^{5}$

\footnotetext{
5. Notice that $\sinh (x)=\frac{\exp (x)-\exp (-x)}{2}$ and thus $\sinh ^{-1}(x)=$ $\ln \left(x+\sqrt{x^{2}+1}\right)=\psi_{U}(x)$.
} 
TABLE 1. Parameters Used in the First Sensitivity Analysis

Domestic risk-free rate, $f(0, t)=f$ for any $t$

0.06

Foreign risk-free rate, $f^{*}(0, t)=f^{*}$ for any $t$

0.05

Commodity drift, $\alpha_{s}$

0.15

Exchange rate drift, $\alpha_{c}$

0.04

Commodity volatility, $\sigma_{s}$

0.15

Exchange rate volatility, $\sigma_{c}$

0.06

Domestic instantaneous forward rate volatility, $\eta_{t, T_{1}}=\eta$ for any $t$ and $T_{1} \quad 0.01$

Foreign instantaneous forward rate volatility, $\eta_{t, T_{2}}^{*}=\eta^{*}$ for any $t$ and $T_{2} \quad 0.01$

Convenience yield volatility, $\sigma_{\delta}$

0.3

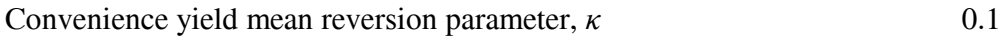

$\begin{array}{ll}\text { Convenience yield long-run mean, } \theta & 0.15\end{array}$

Commodity and exchange rate correlation, $\rho_{1,2} \quad 0.1$

Commodity and domestic instantaneous forward rate correlation, $\rho_{1,3} \quad-0.2$

Commodity and foreign instantaneous forward rate correlation, $\rho_{1,4} \quad-0.25$

Exchange rate and domestic instantaneous forward rate correlation, $\rho_{2,3} \quad 0.05$

Exchange rate and foreign instantaneous forward rate correlation, $\rho_{2,4} \quad 0.1$

Foreign and domestic instantaneous forward rates correlation, $\rho_{3,4} \quad 0.85$

Basket weights (commodity, domestic and foreign zero-coupon bonds) $0.5 ; 0.25 ; 0.25$ Initial values $S_{0}, C_{0}$ and $\delta_{0}$

\section{Performance analysis of Approximations}

In this section, the performance of the three approximations presented previously is analyzed. The prices obtained by Monte Carlo simulations are used as benchmarks since there is no closed-form solution for basket options. Following Barraquand (1995), a variance reduction technique based on the matching of the first and second moments will be used. This will ensure that the sample mean and variance are equal to their theoretical counterparts. The Monte Carlo basket option price combined with the variance reduction technique is given by:

$$
\begin{gathered}
\bar{V}_{0}^{B}=\frac{1}{N} \sum_{i=1}^{N} P(0, T) \max \left(B_{i, T}^{*}-K_{B}, 0\right) \\
\text { where } B_{i, T}^{*}=\left(B_{i, T}-\bar{B}\right) \sqrt{\mu_{2}(v) \hat{S}^{-1}}+\mu_{1}^{\prime}(v),
\end{gathered}
$$

$B_{i, T}$ is the time- $T$ basket value obtained with sample path $i$, 


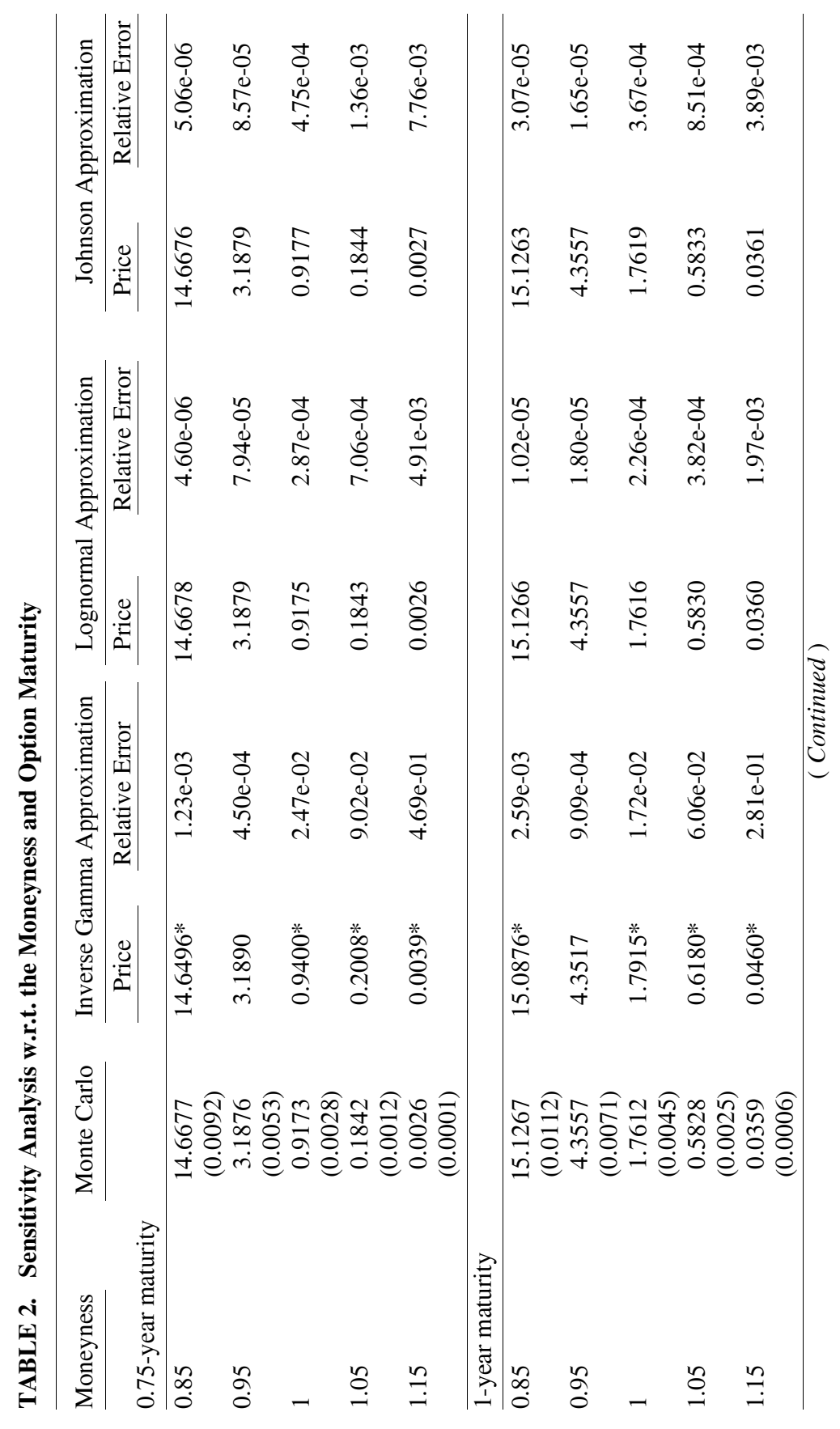




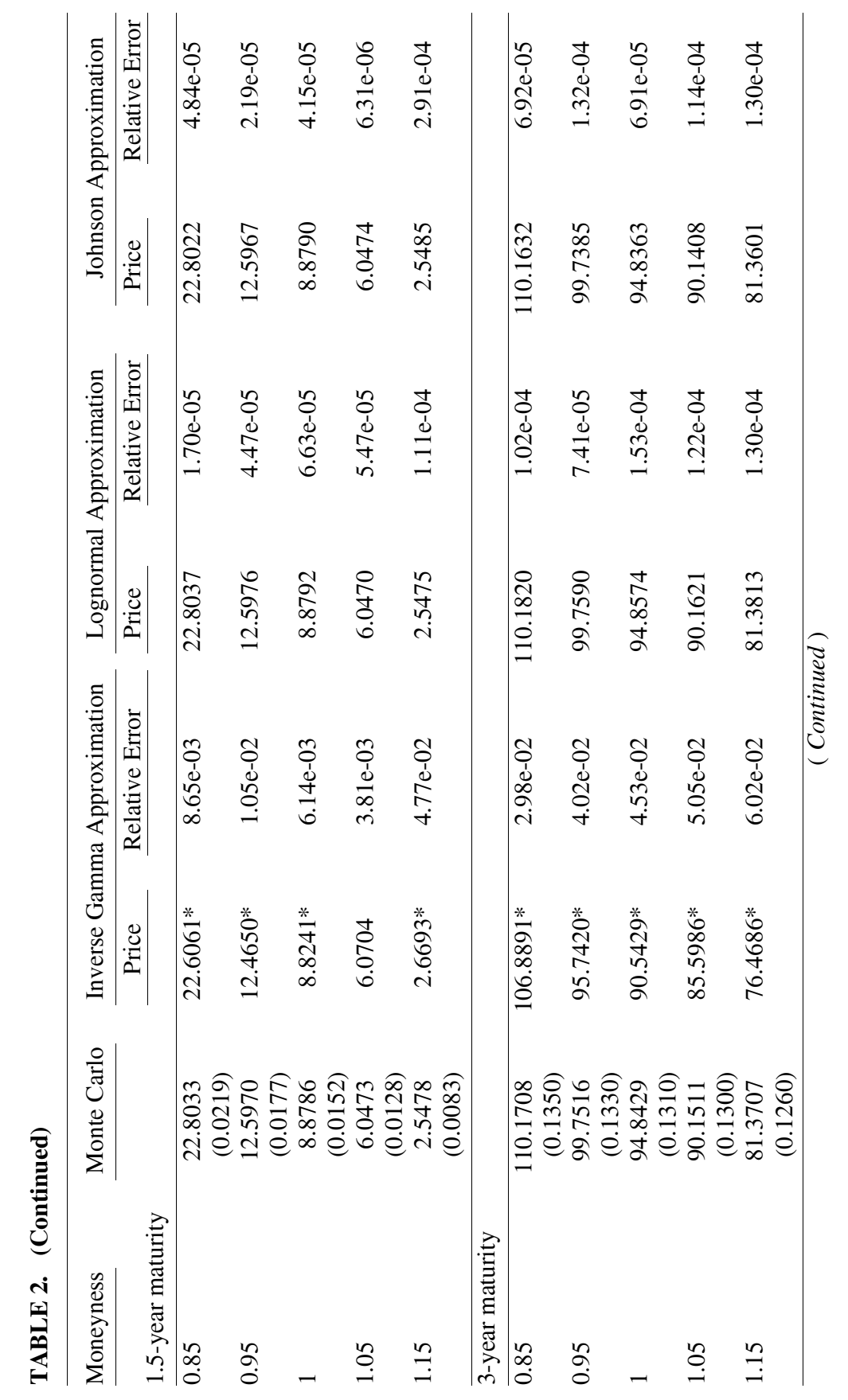




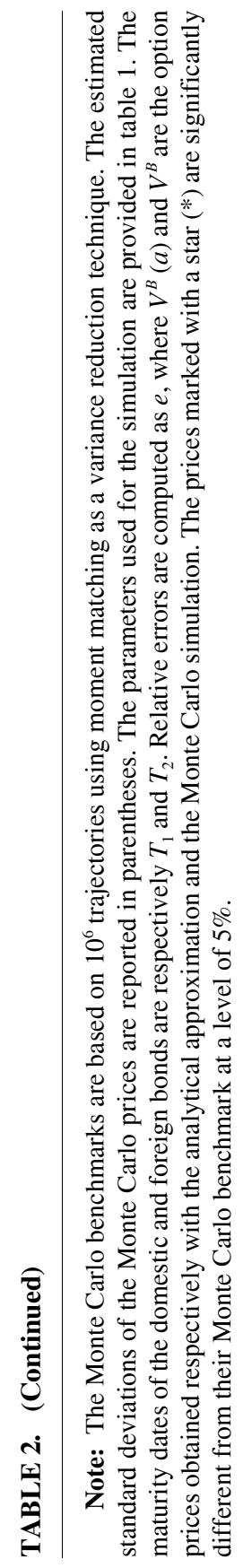




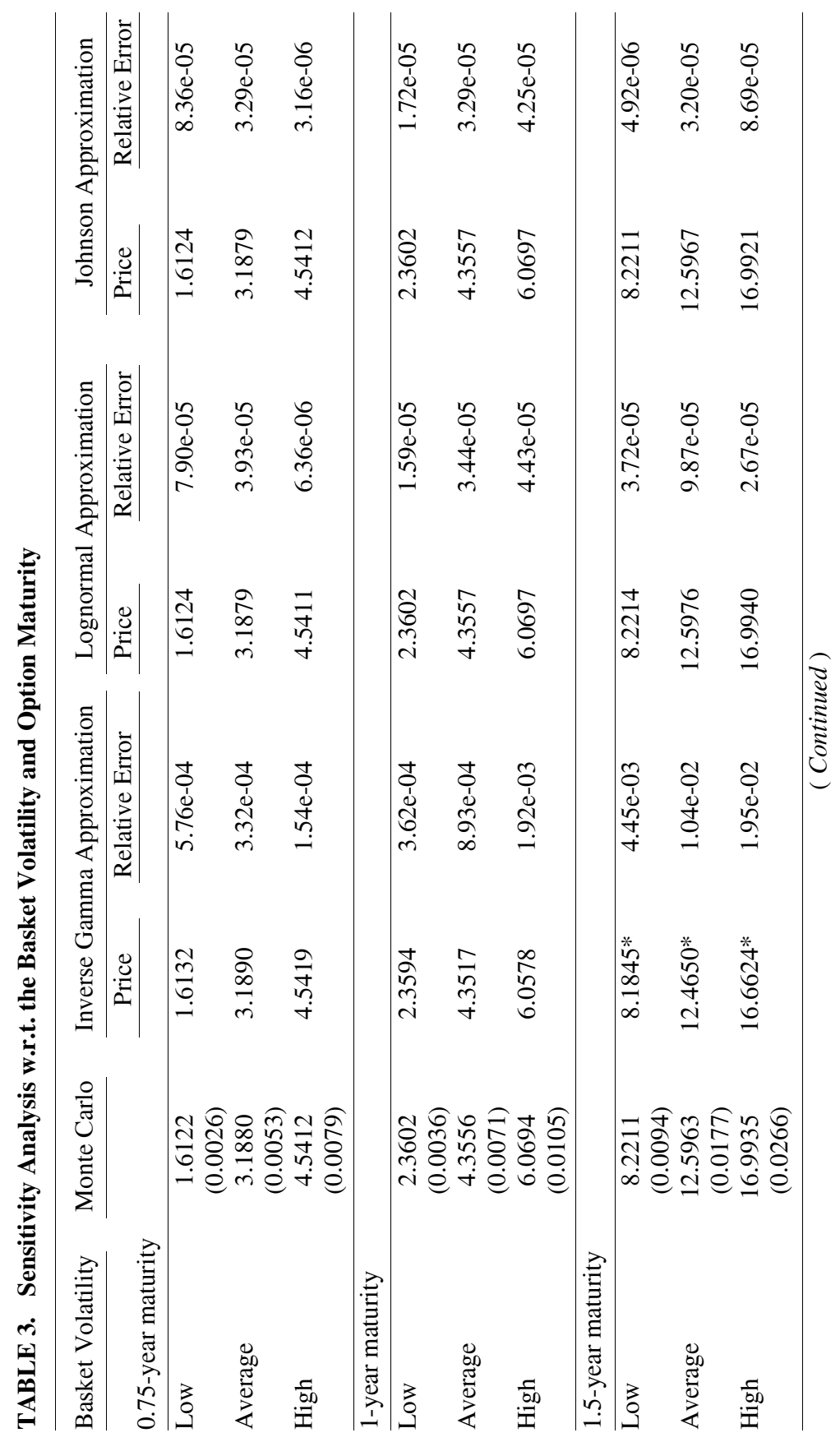




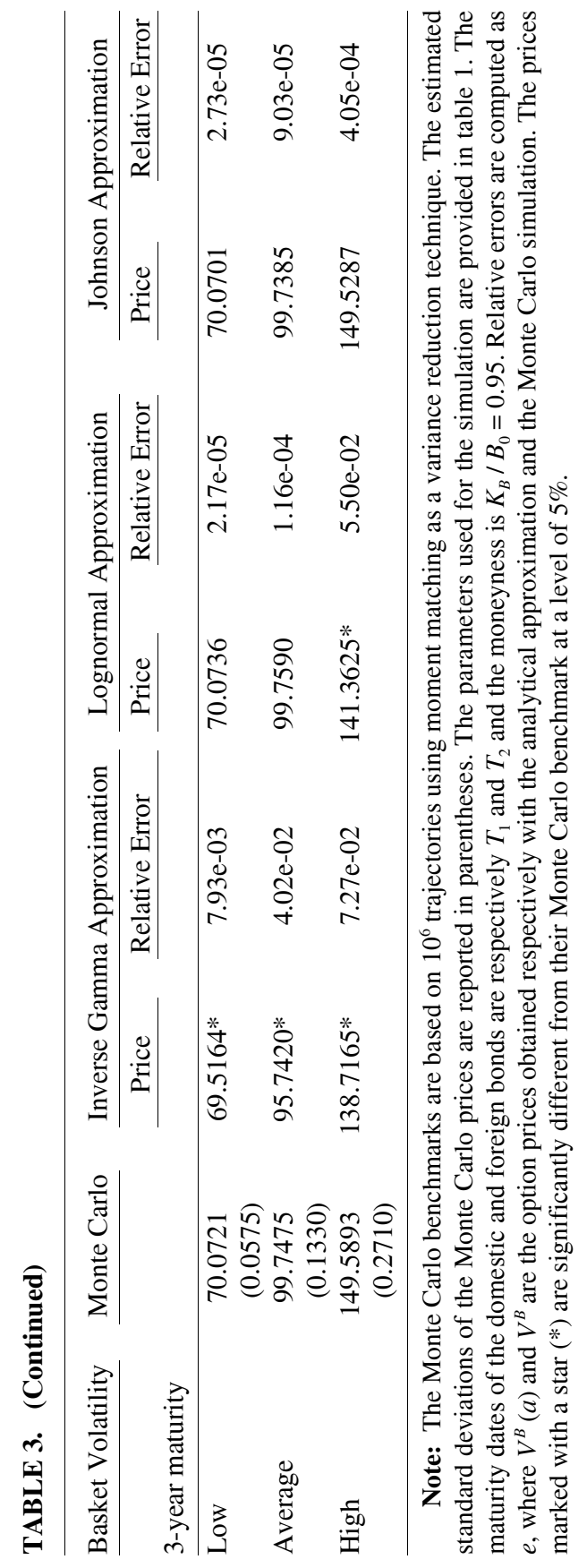


$\bar{B}=\frac{1}{m} \sum_{i=1}^{m} B_{i, T}$ and $\widehat{S}=\sqrt{\frac{1}{m} \sum_{i=1}^{m} B_{i, T}^{2}-\bar{B}^{2}}$ are respectively the sample basket mean and standard deviation and $m$ is the number of simulated paths.

The performance study will be conducted with two analyses. In the first sensitivity analysis, the basket option price obtained with the analytical approximations will be compared to the Monte Carlo price obtained with 1,000,000 paths repeated for different maturities, different moneynesses and different levels for the basket volatility. The second sensitivity analysis is a more detailed analysis based on works done by Broadie and Detemple (1996). It computes the option prices over a wide range of parameters chosen randomly from a realistic set of values in order to generalize the previous results independently of the model's parameters. For each combination, the prices obtained with the approximations and Monte Carlo simulations are compared.

Table 1 presents the set of parameters used in the first analysis. These values are based on estimations using real data on gold prices, USD/CAD exchange rate and Canadian and American 3-month zerocoupon bonds. Although the correlations in the set of parameters take both positive and negative values, the volatility of the basket will increase when individual assets volatilities increase.

Table 2 presents the sensitivity analysis of the basket option price with respect to moneyness, i.e. the ratio of the exercise price to the initial value of the basket $\frac{K_{B}}{B_{0}}$, and option maturity. The results show that Edgeworth-lognormal and Johnson approximations are much more accurate, with relative errors between $10^{-6}$ and $10^{-3}$, than the inverse gamma approximation with a relative error between $10^{-4}$ and $10^{-1}{ }^{6}$

Table 3 presents the sensitivity analysis of the basket option price with respect to the basket volatility and option maturity, for in-the-money options with a moneyness of 0.95 . The average level volatility corresponds to the values in table 1 , while high and low levels correspond respectively to an increase and a decrease of $50 \%$ in the volatility values given in table 1 .

The findings are similar to those in table 2. The relative errors

6. This result is consistent with Milevsky and Posner (1998a) who showed that the convergence result for the inverse gamma works well when the risk-neutral drift of the underlying diffusion is negative or when the correlation matrix decays quickly. 
TABLE 4. Parameters Distributions

Domestic risk-free rate, $f(0, t)=f$ for any $t$

$U([0.02 ; 0.08])$

Foreign risk-free rate, $f^{*}(0, t)=f^{*}$ for any $t$

$U([0.02 ; 0.08])$

Commodity drift, $\alpha_{s}$

Exchange rate drift, $\alpha_{c}$

$U([0.05 ; 0.35])$

Commodity volatility, $\sigma_{s}$

0.04

Exchange rate volatility, $\sigma_{c}$

$U([0.1 ; 0.35])$

Domestic instantaneous forward rate volatility, $\eta_{t, T_{1}}=\eta$

for any $t$ and $T_{1}$

$U([0.02 ; 0.15])$

Foreign instantaneous forward rate volatility, $\eta_{t, T_{2}}^{*}=\eta^{*}$

$U([0.001 ; 0.06])$

for any $t$ and $T_{2}$

Convenience yield volatility, $\sigma_{\delta}$

$U([0.001 ; 0.06])$

Convenience yield mean reversion parameter, $\kappa$

$U([0.1 ; 0.4])$

Convenience yield long-run mean, $\theta$

$U([0.05 ; 0.8])$

Commodity and exchange rate correlation, $\rho_{1,2}$

$U([0.05 ; 0.5])$

$U([0.01 ; 0.55])$

Commodity and domestic instantaneous forward rate correlation, $\rho_{1,3} U([-0.5 ; 0.25])$

Commodity and foreign instantaneous forward rate correlation, $\rho_{1,4} \quad U([-0.5 ; 0.25])$

Exchange rate and domestic instantaneous forward rate correlation, $\rho_{2,3}$

$U([-0.35 ; 0.35])$

Exchange rate and foreign instantaneous forward rate correlation, $\rho_{2,4}$

$U([-0.35 ; 0.35])$

Foreign and domestic instantaneous forward rates correlation, $\rho_{3,4}$

Option maturity (in years)

$U([0.15 ; 0.9])$

$U(\{0.083 ; 0.25$;

Moneyness

$0.5 ; 0.75 ; 1 ; 1.5 ; 2 ; 3\})$

$U(\{0.8 ; 0.9 ; 0.95$;

$1 ; 1.05 ; 1.1 ; 1.2\})$

Basket weights (commodity, domestic and foreign zero-coupon bonds)

$$
U\left(\begin{array}{l}
\left(\frac{1}{3}, \frac{1}{3}, \frac{1}{3},\right) ;\left(\frac{1}{2}, \frac{1}{4}, \frac{1}{4}\right) ; \\
\left(\frac{1}{4}, \frac{1}{2}, \frac{1}{4},\right) ;\left(\frac{1}{4}, \frac{1}{4}, \frac{1}{2}\right)
\end{array}\right)
$$

Initial Values $S_{0}, C_{0}$ and $\delta_{0}$ \$330; \$/CAD 0.65; 0.2

presented in table 3 are very low with a magnitude between $10^{-6}$ and $10^{-2}$. For low volatility levels, Edgeworth-lognormal and Johnson approximate prices and Monte Carlo prices are very close. As for the inverse gamma approximation, the results show that it is much less accurate with relative errors between $10^{-4}$ and $10^{-2}$. Note that the accuracy of the three approximations decreases for longer maturities and higher volatilities, which supports the findings in $\mathrm{Ju}(2002){ }^{7}$

7. $\mathrm{Ju}(2002)$ proposes an analytical approximation to price Asian and basket options 
TABLE 5. RMSE and MRE for the Three Approximations

\begin{tabular}{lccc}
\hline & $\begin{array}{r}\text { Inverse Gamma } \\
\text { Approximation }\end{array}$ & $\begin{array}{c}\text { Lognormal } \\
\text { Approximation }\end{array}$ & $\begin{array}{c}\text { Johnson } \\
\text { Approximation }\end{array}$ \\
\hline RMSE & $9.68 \%$ & $0.52 \%$ & $0.56 \%$ \\
MRE & $79.47 \%$ & $13.16 \%$ & $14.00 \%$ \\
\hline
\end{tabular}

Moreover, for all approximations, option prices increase with maturity and volatility.

The previous analysis is only local and the findings depend on the set of parameters used and may change if the parameters values are modified. To confirm these conclusions, a more carefully designed numerical study where the parameters are randomly chosen is conducted. Following Broadie and Detemple (1996), the significant model parameters are chosen randomly from continuous and discrete uniform distributions. These uniform distributions are based on the estimation of the model parameters using real data. The correlation between the commodity and the exchange rate is expected to be positive, and so it is assumed that $\rho_{12} \in[0.01,0.55]$. Based on Schwartz (1997), the convenience yield long-run mean is positive for gold and the mean reversion parameter is small and less than 1 ; it is therefore assumed that $\theta \in[0.05,0.5]$ and $\kappa \in[0.05,0.8]$. All parameter distributions used in the pricing are presented in table 4.

The three previous analytical approximations and Monte Carlo simulation combined with the variance reduction technique are compared for 5,000 random sets of parameters. Only 4,347 sets of parameters are left after non-positive definite correlation matrices and basket option prices lower than 5 cents are removed from the sample. First, the accuracy of each approximation is examined by calculating its root mean squared error (RMSE). Second, the maximum relative error (MRE) is computed for each approximation to examine the worst case scenario. More precisely, we define,

based on a Taylor expansion of the ratio of the characteristic function of the average of lognormal variables to that of the approximating lognormal random variable around zero volatility. 


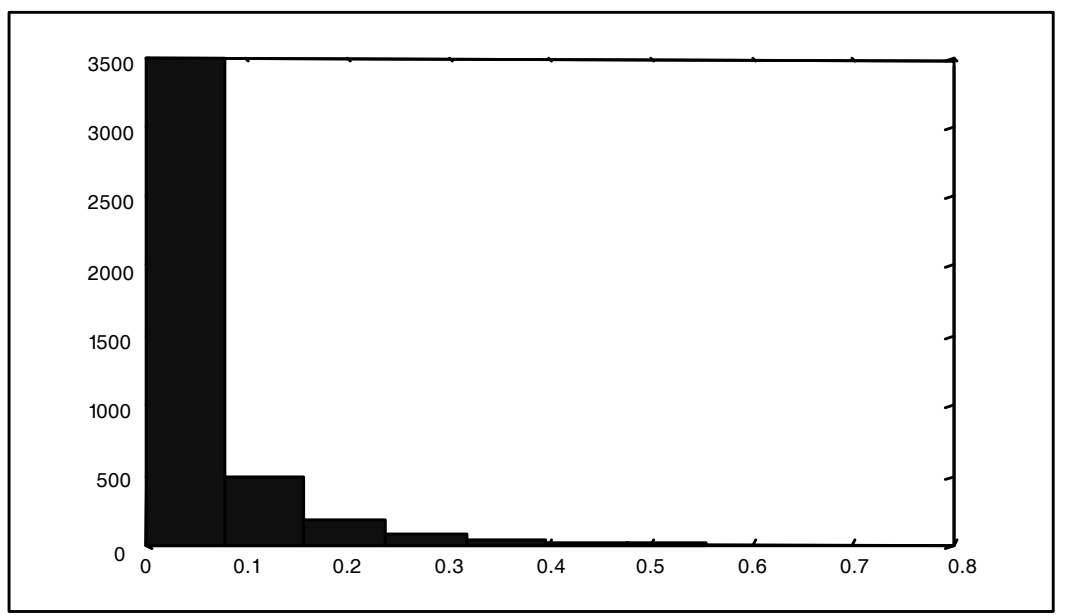

$\mathrm{X}$-axis represents relative errors

Y-axis represents the number of observations

FIGURE 1.- Histogram of Relative Errors of the Inverse Gamma Approximation

$$
\begin{gathered}
R M S E=\sqrt{\frac{1}{n} \sum_{i=1}^{n}\left(\frac{V_{i}^{B}(a)-\bar{V}_{i}^{B}}{\bar{V}_{i}^{B}}\right)^{2}} \\
M R E=\max _{i} \frac{\left|V_{i}^{B}(a)-\bar{V}_{i}^{B}\right|}{\bar{V}_{i}^{B}},
\end{gathered}
$$

where $n=4,347$ is the number of the different sets of parameters, and $V_{i}^{B}(a)$ and $\bar{V}_{i}^{B}$ correspond respectively to the approximate and Monte Carlo prices for parameters set $i$. Monte Carlo prices are obtained with $1,000,000$ paths combined with the variance reduction technique.

The results in table 5 confirm those obtained with the first sensitivity analysis (tables 2 and 3 ) and show that the Edgeworth-lognormal and Johnson approximations are much more accurate than the inverse gamma approximation. It is also found that, for the Edgeworth-lognormal and Johnson approximations, a very small 


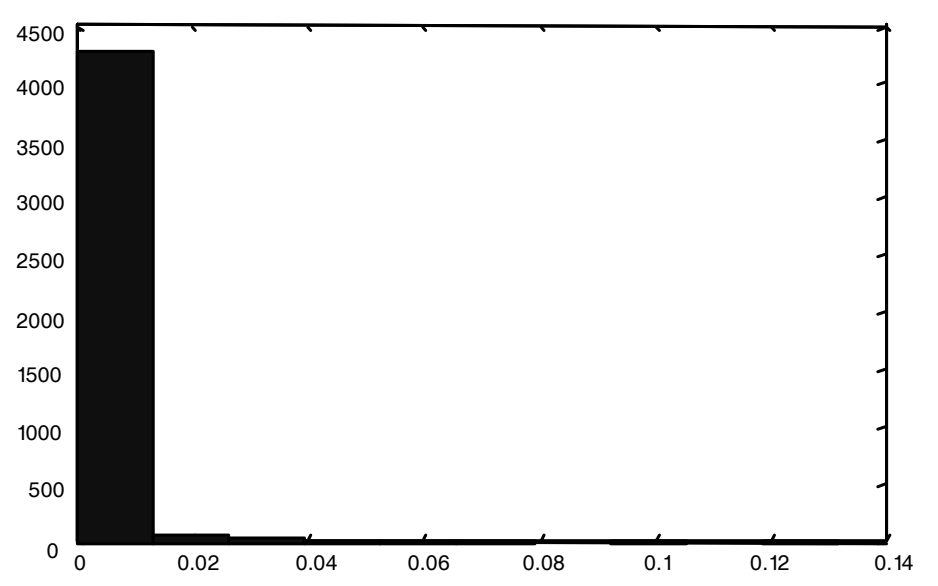

$\mathrm{X}$-axis represents relative errors

$\mathrm{Y}$-axis represents the number of observations

FIGURE 2.- Histogram of Relative Errors of the Edgeworth Approximation

proportion of options, $0.23 \%$ and $0.35 \%$ respectively, have relative errors above 5\% while for the inverse gamma approximation a larger proportion, $27.74 \%$, of options have a relative error above $5 \%$.

A more detailed look at the results shows that out-of-the-money and high volatility options have the largest relative errors, which confirms our findings in the first sensitivity analysis. However, since the out-of-the-money options have small prices, the augmentation of the relative errors in these cases may be attributed to the small denominators. Figures 1, 2 and 3 present respectively the histograms of the relative errors for the inverse gamma, the Edgeworth-lognormal and Johnson approximations. Table 6 shows a summary of descriptive statistics of the relative errors.

The histograms show that for the Edgeworth-lognormal and Johnson approximations, $99 \%$ of parameters sets $(4,300$ out of 4,347$)$ have relative errors less than $2 \%$. This demonstrates that these approximations are very accurate and that they give prices very close to 


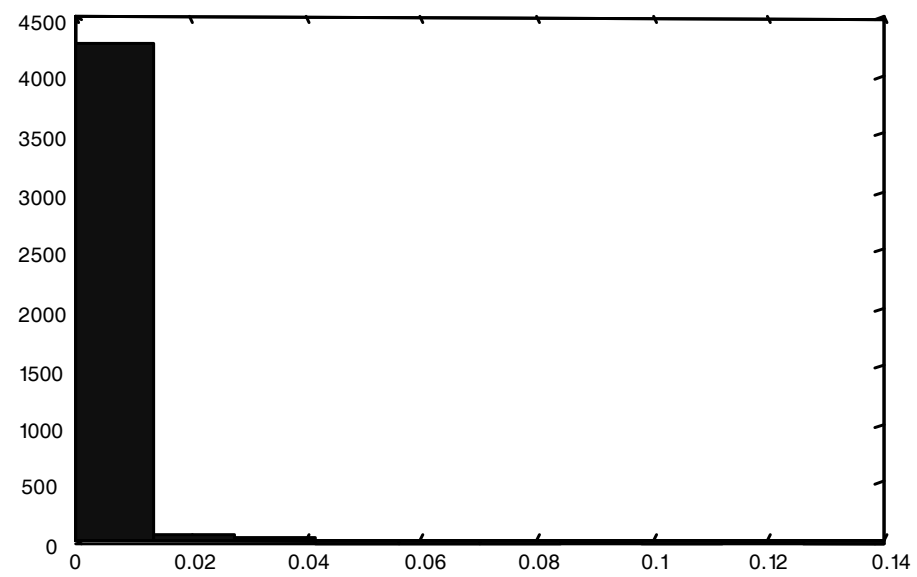

$\mathrm{X}$-axis represents relative errors

$\mathrm{Y}$-axis represents the number of observations

FIGURE 3.- Histogram of Relative Errors of Johnson Approximation

Monte Carlo benchmarks. For the inverse gamma approximation, 92\% $(4,000$ out of 4,347$)$ of the cases have relative errors less than $20 \%$. The same results are found by using larger and more general intervals for the parameters distributions.

To summarize this section regarding the pricing of heterogeneous basket options, it is found that the Edgeworth expansion around the lognormal distribution at order 4 and the Johnson distribution are equally accurate and very acceptable to practitioners. However, the use of the Edgeworth-lognormal distribution may be preferred for two reasons: first, it is slightly more accurate, and second, the algorithm to calibrate Johnson distributions may not converge in a few cases, which can lead to mispriced options. 
TABLE 6. Descriptive Statistics of Relative Errors

\begin{tabular}{lccc}
\hline & $\begin{array}{c}\text { Inverse Gamma } \\
\text { Approximation }\end{array}$ & $\begin{array}{c}\text { Lognormal } \\
\text { Approximation }\end{array}$ & $\begin{array}{c}\text { Johnson } \\
\text { Approximation }\end{array}$ \\
\hline Maximum & 0.7947 & 0.1316 & 0.1400 \\
Minimum & 0 & 0 & 0 \\
Mean & 0.0493 & 0.0015 & 0.0014 \\
Median & 0.0136 & 0.0002 & 0.0001 \\
Standard Deviation & 0.0833 & 0.0050 & 0.0054 \\
Nb of Observations & 4347 & 4347 & 4347 \\
\hline
\end{tabular}

\section{Extensions}

\section{A. Hedging Ratios}

The analytical approximations allow for deriving the option price in a functional form which can also provide analytical expressions for the sensitivities with respect to the underlying parameters, such as deltas, vegas and theta, known as the hedging ratios or the Greeks. However, due to the complexity of the moments involved in our analytical approximations, deriving the hedging ratios analytically is beyond the scope of this article. Instead, they can be computed numerically as follows:

$$
\operatorname{Ratio}_{A p p}^{B}=\frac{V_{A p p}^{B(\varepsilon)}-V_{A p p}^{B}}{\varepsilon}
$$

where $\varepsilon>0$ is a small number, $V_{A p p}^{B(\varepsilon)}$ and $V_{A p p}^{B}$ are respectively the approximate $\varepsilon$-disturbed and non-disturbed option prices. As an application, the delta with respect to the commodity price is computed numerically for different values of $\varepsilon$. Table 7 presents the calculations for a 9-month in-the-money call basket option (moneyness 0.95) using the parameters in table 1 .

It is shown that for the three approximations, the delta is very stable over different values of $\varepsilon$. Indeed, an increase of $\$ 1$ in the commodity price leads to an increase of approximately $\$ 0.01$ in the option price. The positiveness of delta is expected but its value depends on the set of parameters used. Other sensitivities with respect to other parameters of interest can be computed with the same procedure. 
TABLE 7. Basket Option Delta w.r.t. the Commodity Price

\begin{tabular}{|c|c|c|c|c|c|c|}
\hline \multirow[t]{2}{*}{$\begin{array}{c}\text { Commodity } \\
\text { Price }\end{array}$} & \multicolumn{2}{|c|}{$\begin{array}{l}\text { Inverse Gamma } \\
\text { Approximation }\end{array}$} & \multicolumn{2}{|c|}{$\begin{array}{c}\text { Lognormal } \\
\text { Approximation }\end{array}$} & \multicolumn{2}{|c|}{$\begin{array}{c}\text { Johnson } \\
\text { Approximation }\end{array}$} \\
\hline & Price & Delta* & Price & Delta* & Price & Delta* \\
\hline$\$ 330$ & 3.1890 & & 3.1879 & & 3.1879 & \\
\hline$\$ 330.33$ & 3.1922 & 0.0097 & 3.1910 & 0.0094 & 3.1911 & 0.0097 \\
\hline$\$ 331.65$ & 3.2049 & 0.0096 & 3.2037 & 0.0096 & 3.2038 & 0.0096 \\
\hline$\$ 333.30$ & 3.2208 & 0.0096 & 3.2196 & 0.0096 & 3.2196 & 0.0096 \\
\hline$\$ 334.95$ & 3.2367 & 0.0096 & 3.2355 & 0.0096 & 3.2355 & 0.0096 \\
\hline
\end{tabular}

Note: Delta corresponds to the sensitivity with respect to the commodity price and is given by equation (14). $V_{A p p}^{B}$, presented in the first row of the table, is the analytical basket option price obtained for a commodity price of $\$ 330$

\section{B. Stochastic Volatility}

Many recent empirical studies show that models incorporating stochastic volatility and jumps capture some well-known stylized facts, such as volatility smile, fat tails, and large moves (see Heston (1993), Bakshi, Cao and Chen (1997), and Bakshi, Carr and Wu (2006)). However, the high-dimensionality and the heterogeneous character of this model makes it difficult to extend it to a more general and flexible framework. The following are some thoughts on the various extensions that can refine the model to account for these empirical findings in option pricing literature.

In this model, the basket price depends on four correlated assets modeled with five diffusion processes, namely the commodity price and its convenience yield, the exchange rate, the domestic and the foreign zero-coupon bonds. Adding very general stochastic volatility/jump processes will obviously make the model intractable and not parsimonious. It will certainly be impossible to derive similar analytical approximations as the basket moments cannot be obtained in closed-form solutions. However, if we assume that the stochastic volatility processes are not correlated with their corresponding assets and with each other, ${ }^{8}$ we can assume a multi-dimensional version of the Hull and White (1987) stochastic volatility model or the multivariate Wishart process in Gourieroux and Sufana (2004) to price the proposed

8. Many empirical studies show that the volatility smile is symmetric in Forex and interest rates markets and thus the correlation between the volatility and these types of assets can be considered as zero. 
heterogeneous basket option. ${ }^{9}$ More precisely, the density function of each of the basket components can be approximated by conditioning on its volatility path. In doing so, a stochastic volatility diffusion can be replaced by a constant volatility diffusion where the constant diffusion parameter is given as the square root of the average variance over the option's life (see Sabanis (2003) and Tahani (2005)). The basket moments under the multi-dimensional stochastic volatility framework can be approximated and therefore the analytical moment-matching approximations derived. These approximations under a stochastic volatility framework do not necessarily perform as well as under the constant volatility framework; and need to be assessed using a robust numerical analysis.

\section{Implied Volatility}

A recent paper by Bakshi and Madan (2006) analyses the difference between physical and risk-neutral volatilities, known as volatility spreads. Using the S\&P 100 index options the authors confirm empirically what the theory predicts: volatility spreads should be positive. In this setting and assuming that basket option prices are available from the market, we can easily use the analytical approximations to infer the risk-neutral model parameters by minimizing the quadratic error between the theoretical and the market prices. This calibration should be much faster with the analytical approximations than with Monte Carlo simulations or any other numerical technique such as finite differences. On the other hand, the physical model parameters can be simply inferred from the prices of the assets underlying the basket, i.e. the commodity, the exchange rate, and the interest rates. Finally, once the parameters are estimated under both the physical and the risk-neutral measures, one can easily analyze the differences between the physical and the risk-neutral basket volatilities and skewness in light of Bakshi and Madan (2006) work.

\section{Optimal Portfolio Selection}

The analytical approximations derived under the $T$-forward measure $Q_{T}$ for the purpose of basket options pricing can be adapted to derive the optimal portfolio, i.e. the optimal numbers of shares invested in each of the basket components. Indeed, using the appropriate moments for the

9. Note that some jumps may need to be added. 
basket value under the physical measure $P$ and assuming that the approximate densities will still perform well under this measure, one can approximate the expected utility of the basket. ${ }^{10}$ Using the Johnson distribution for example, we have

$$
E^{P}\left[U\left(B_{T}\right)\right]=\int_{-\infty}^{+\infty} U\left(\varepsilon+\lambda \psi^{-1}\left(\frac{z-\gamma}{\delta}\right)\right) \frac{1}{\sqrt{2 \pi}} \exp \left(-\frac{z^{2}}{2}\right) d z
$$

where $U(\bullet)$ is the utility function and $\psi(\bullet)$ is defined in equations (10). Depending on the choice of $U$ and $\psi$, this expectation can be computed analytically, yielding an expression that will depend on the utility parameters and Johnson parameters $(\varepsilon, \lambda, \gamma, \delta)$. Since Johnson parameters are obtained by matching the first four moments of the basket, the expected utility can be seen as a highly non-linear function of the numbers of shares $w_{1}, w_{2}$ and $w_{3}$. Although, in practice, these weights are usually determined by the firm's production, its debt structure, and its liquidity needs, it can be interesting to obtain them by maximizing the expected utility $E^{P}\left[U\left(B_{T}\right)\right]$. This optimization will most likely be performed numerically and may be subject to many local optima. Note also that the optimal weights will depend on the choice of the utility function.

\section{Conclusion}

Firms can use basket options to hedge their exposure to different risks, such as commodity risk, interest rate risk and exchange rate risk. However, pricing this kind of options is not an easy task since no closed-form solution can be derived for the basket density function. Consequently, a standard pricing formula such as Black and Scholes cannot be derived. The main contribution of this article is the comparison of the performance of three analytical approximations to price a heterogeneous basket option, consisting of a commodity, a domestic and a foreign zero-coupon bonds, when interest rates are stochastic. The three approximations used are the inverse gamma proposed in Milevsky and Posner (1998a, 1998b), the Edgeworth expansion around the lognormal distribution as well as Johnson

10. The best approximate density will be assessed numerically using Monte Carlo simulations for a large pool of parameters 
distribution developed in Posner and Milevsky (1999).

In order to assess and compare the accuracy of the approximations, two analyses are conducted. The first one is a local sensitivity analysis where the parameters of the model are fixed arbitrarily. The second one is based on a random choice of parameters. The findings show that both the Edgeworth-lognormal and Johnson approximations are very accurate while the inverse gamma approximation is much less accurate.

Accepted by: Prof. P. Theodossiou, Editor-in-Chief, January 2008

\section{Appendices}

\section{A. Derivation of the Forward Measure}

In this appendix, we will derive the model under the $T$-forward measure. Let $\mathrm{A}_{i}$ be the $i^{\text {th }}$ row of the matrix A, where $\mathrm{A}=\left(a_{i j}\right)_{i, j=\{1,2,3,4\}}$ is the Cholesky decomposition of the correlation matrix of the fourdimensional $P$-Brownian motion $W=\left(W^{(1)}, W^{(2)}, W^{(3)}, W^{(4)}\right)^{\prime}$, and $\tilde{\mathrm{B}}$ corresponds to the vector of independent Brownian motions under $Q$. The SDEs satisfied by all underlying assets under $Q$ can be written as: ${ }^{11}$

$$
\begin{gathered}
d S_{t}=S_{t}\left[\left(r_{t}-\delta_{t}\right) d t+\sigma_{s} \mathrm{~A}_{1} d \tilde{\mathrm{B}}_{t}\right] \\
d \delta_{t}=\left(\kappa \theta-\frac{\sigma_{\delta}}{\sigma_{s}}\left(\alpha_{s}-r_{t}\right)-\kappa \delta_{t}\right) d t+\sigma_{\delta} \mathrm{A}_{1} d \tilde{\mathrm{B}}_{t} \\
d C_{t}=C_{t}\left[\left(r_{t}-r_{t}^{*}\right) d t+\sigma_{c} \mathrm{~A}_{2} d \tilde{\mathrm{B}}_{t}\right] \\
d P\left(t, T_{1}\right)=P\left(t, T_{1}\right)\left[r_{t} d t-\beta_{t, T_{1}} \mathrm{~A}_{4} d \tilde{\mathrm{B}}_{t}\right] \\
d P^{*}\left(t, T_{2}\right)=P^{*}\left(t, T_{2}\right)\left[\left(r_{t}^{*}+\beta_{t, T_{2}}^{*} \sigma_{c} \rho_{2,4}\right) d t-\beta_{t, T_{2}}^{*} \mathrm{~A}_{4} d \tilde{\mathrm{B}}_{t}\right]
\end{gathered}
$$

\footnotetext{
11. The details of the computation are available from the authors upon request.
} 
The risk-neutral $Q$ corresponds to a numeraire equal to the domestic bank account $\exp \left(\int_{t}^{T} r_{u} d u\right)$, while the $T$-forward measure $Q_{T}$ corresponds to a numeraire equal to the domestic zero-coupon bond with maturity date $T, P(t, T)$. Using Girsanov theorem, we have that:

$$
d \hat{\mathrm{B}}_{t}=d \tilde{\mathrm{B}}_{t}+\beta_{t, T} \mathrm{~A}_{3}^{\prime} d t
$$

where $\hat{\mathrm{B}}$ are independent Brownian motions under $Q_{T}$. The correlation structure is the same under $Q$ and $Q_{T}$, and the previous SDE's can be rewritten as:

$$
\begin{gathered}
d S_{t}=S_{t}\left\lfloor\left(r_{t}-\delta_{t}-\rho_{1,3} \sigma_{s} \beta_{t, T}\right) d t+\sigma_{s} d \hat{W}_{t}^{(1)}\right\rfloor \\
d \delta_{t}=\left(\kappa \theta-\frac{\sigma_{\delta}}{\sigma_{s}}\left(\alpha_{s}-r_{t}\right)-\kappa \delta_{t}-\rho_{1,3} \sigma_{\delta} \beta_{t, T}\right) d t+\sigma_{\delta} d \hat{W}_{t}^{(1)} \\
d C_{t}=C_{t}\left\lfloor\left(r_{t}-r_{t}^{*}-\rho_{2,3} \sigma_{c} \beta_{t, T}\right) d t+\sigma_{c} d \hat{W}_{t}^{(2)}\right\rfloor \\
d P\left(t, T_{1}\right)=P\left(t, T_{1}\right)\left\lfloor\left(r_{t}+\beta_{t, T_{1}} \beta_{t, T}\right) d t-\beta_{t, T_{1}} d \hat{W}_{t}^{(3)}\right\rfloor \\
d P^{*}\left(t, T_{2}\right)=P^{*}\left(t, T_{2}\right)\left\lfloor\left(r_{t}^{*}+\beta_{t, T_{2}}^{*} \sigma_{c} \rho_{2,4}+\rho_{3,4} \beta_{t, T} \beta_{t, T_{2}}^{*}\right) d t-\beta_{t, T_{2}}^{*} d \hat{W}_{t}^{(4)}\right\rfloor
\end{gathered}
$$

where $\widehat{W}=\left(\hat{W}^{(1)}, \hat{W}^{(2)}, \hat{W}^{(3)}, \hat{W}^{(4)}\right)^{\prime}=A \hat{B}$. The strong solution exists and is given by the system equation (3).

\section{B. Derivation of Moments}

This appendix gives the detailed calculation of the first four moments of the basket value at maturity $T$ under the $T$-forward measure $Q_{T}$. Using the strong solution of the model under the forward-measure $Q_{T}$, one can write: 


$$
\begin{gathered}
S_{T}=S_{0} \exp \left(\phi_{s}(T)+\sum_{i=1}^{4} \int_{0}^{T} \psi_{S, i}(t) d \hat{W}_{t}^{(i)}\right) \\
P\left(T, T_{1}\right)=\exp \left(\phi_{P}(T)+\sum_{i=1}^{4} \int_{0}^{T} \psi_{P, i}(t) d \hat{W}_{t}^{(i)}\right) \\
C_{T} P^{*}\left(T, T_{2}\right)=C_{0} \exp \left(\phi_{C P^{*}}(T)+\sum_{i=1}^{4} \int_{0}^{T} \psi_{C P^{*}, i}(t) d \hat{W}_{t}^{(i)}\right)
\end{gathered}
$$

where

$$
\begin{aligned}
& \phi_{S}(T)=\left[\begin{array}{l}
\int_{0}^{T}\left(1-\frac{\sigma_{\delta}}{\sigma_{s} \kappa}\left(1-e^{-\kappa(T-u)}\right)\right) f(0, u) d u-\delta_{0} \frac{1}{\kappa}\left(1-e^{-\kappa T}\right) \\
-\left(\theta-\frac{\sigma_{\delta}}{\sigma_{s}} \frac{\alpha_{s}}{\kappa}+\frac{\sigma_{s}^{2}}{2}\right) T+\left(\theta-\frac{\sigma_{\delta}}{\sigma_{s}} \frac{\alpha_{s}}{\kappa}\right) \frac{1}{\kappa}\left(1-e^{-\kappa T}\right) \\
-\frac{1}{2} \int_{0}^{T} \beta_{u, T}^{2} d u+\frac{\sigma_{\delta}}{\sigma_{s} \kappa} \int_{0}^{T}\left(1-e^{-\kappa(T-u)}\right)\left(\int_{0}^{u} \eta_{v, u}\left(\beta_{v, T}-\beta_{v, u}\right) d v\right) d u \\
+\rho_{1,3} \int_{0}^{T} \beta_{u, T}\left(\frac{\sigma_{\delta}}{\kappa}\left(1-e^{-\kappa(T-u)}\right)-\sigma_{s}\right) d u
\end{array}\right], \\
& \phi_{P}(T)=-\int_{T}^{T_{1}} f(0, u) d u-\frac{1}{2} \int_{0}^{T}\left(\beta_{u, T_{1}}-\beta_{u, T}\right)^{2} d u \\
& \phi_{C P^{*}}(T)=\int_{0}^{T} f(0, u) d u-\int_{0}^{T_{2}} f^{*}(0, u) d u-\frac{1}{2} \int_{0}^{T}\left(\beta_{u, T}^{2}+\left(\beta_{u, T_{2}}^{*}\right)^{2}\right) d u \\
& -\frac{\sigma_{c}^{2}}{2} T-\sigma_{c} \rho_{2,3} \int_{0}^{T} \beta_{u, T} d u+\sigma_{c} \rho_{2,4} \int_{0}^{T} \beta_{u, T_{2}}^{*} d u \\
& +\rho_{3,4} \int_{0}^{T} \beta_{u, T_{2}}^{*} \beta_{u, T} d u
\end{aligned}
$$




$$
\begin{gathered}
\psi_{S, 1}(t)=\sigma_{s}-\frac{\sigma_{\delta}}{\kappa}\left(1-e^{-\kappa(T-t)}\right), \\
\psi_{S, 3}(t)=\beta_{t, T}-\frac{\sigma_{\delta}}{\sigma_{s} \kappa} \int_{t}^{T}\left(1-e^{-\kappa(T-v)}\right) \eta_{t, v} d v \\
\psi_{P, 3}(t)=\beta_{t, T}-\beta_{t, T_{1}}, \\
\psi_{C P^{*}, 2}(t)=\sigma_{c}, \\
\psi_{C P^{*}, 3}(t)=\beta_{t, T}, \\
\psi_{C P^{*}, 4}(t)=-\beta_{t, T_{2}}^{*}, \\
\psi_{S, 2}(t)=\psi_{S, 4}(t)=\psi_{P, 1}(t)=\psi_{P, 2}(t)=\psi_{P, 4}(t)=\psi_{C P^{*}, 1}(t)=0 .
\end{gathered}
$$

Therefore,

$$
\begin{aligned}
\mu_{n}^{\prime}= & \sum_{k=0}^{n} \sum_{j=0}^{k} \frac{n !}{j !(k-j) !(n-k) !} w_{1}^{j} w_{2}^{(k-j)} w_{3}^{(n-k)} E^{Q_{T}}\left[S_{T}^{j}\left(P\left(T, T_{1}\right)\right)^{k-j}\right. \\
& \left.C_{T}^{n-k}\left(P^{*}\left(T, T_{2}\right)\right)^{n-k}\right]
\end{aligned}
$$

with

$$
\begin{gathered}
E^{Q_{T}}\left[S_{T}^{j}\left(P\left(T, T_{1}\right)\right)^{k-j} C_{T}^{n-k}\left(P^{*}\left(T, T_{2}\right)\right)^{n-k}\right\rfloor \\
=S_{0}^{j} C_{0}^{n-k}\left(P^{*}\left(0, T_{2}\right)\right)^{n-k} E^{Q_{T}}\left[\exp \left(\phi_{j k}(T)+\sum_{i=1}^{4} \int_{0}^{T} \psi_{i j k}(t) d \hat{W}_{t}^{i}\right)\right] \\
=S_{0}^{j} C_{0}^{n-k}\left(P^{*}\left(0, T_{2}\right)\right)^{n-k} \exp \left(\phi_{j k}(T)+\frac{1}{2} \sum_{i=1}^{4} \sum_{l=1}^{4} \rho_{i, l} \int_{0}^{T} \psi_{i j k}(t) \psi_{l j k}(t) d t\right)
\end{gathered}
$$


TABLE 8. Comparison of Theoretical and Simulated Moments

\begin{tabular}{lcc}
\hline Order & Theoretical Moments & Simulated Moments \\
\hline 1 & 157.21 & 157.20 \\
2 & 24807 & 24807 \\
3 & $3.9293 \mathrm{e}+6$ & $3.9292 \mathrm{e}+6$ \\
4 & $6.2472 \mathrm{e}+8$ & $6.2470 \mathrm{e}+8$ \\
Mean & 157.21 & 157.20 \\
Variance & 93.13 & 93.13 \\
\hline
\end{tabular}

where

$$
\begin{gathered}
\phi_{j k}(T)=j \phi_{S}(T)+(k-j) \phi_{P}(T)+(n-k) \phi_{P^{*}}(T) \\
\psi_{i j k}(t)=j \psi_{S, i}(t)+(k-j) \psi_{P, i}(t)+(n-k) \psi_{P^{*}, i}(t), i \in\{1,2,3,4\} .
\end{gathered}
$$

Table 8 presents the theoretical first four moments, calculated as explained previously, and those obtained by Monte Carlo simulation. This ensures that our theoretical formulas give the exact moments.

\section{Inverse Gamma Approximation}

This appendix shows how we obtain the pricing formula using the inverse gamma approximation. The density function of a gamma random variable $X$ of parameters $(\alpha, \beta), X \sim G(\alpha, \beta)$, is given by:

$$
g(x \mid \alpha, \beta)=\frac{x^{\alpha-1} \exp \left(-\frac{x}{\beta}\right)}{\beta^{\alpha} \Gamma(a)}, x>0
$$

where $\alpha>0, \beta>0$ and $\Gamma(\alpha)$ is the Gamma function.

Proposition 1: Let $X$ be a gamma random variable with parameters $(\alpha, \beta)$. Then, the random variable $Y=\frac{1}{X}$ follows an inverse gamma distribution, $Y \sim G_{R}(\alpha, \beta)$, and its density function is given by:

$$
g_{R}(y \mid \alpha, \beta)=\frac{1}{y^{2}} g\left(\frac{1}{y} \mid \alpha, \beta\right)=\frac{\exp \left(-\frac{1}{y \beta}\right)}{y^{\alpha+1} \beta^{\alpha} \Gamma(a)}, y>0, \alpha, \beta>0
$$


Proposition 2: The non-centered moments of the random variable $Y \sim G_{R}(\alpha, \beta)$ are given by:

$$
\mu_{n}^{\prime}\left(g_{R}\right)=\frac{1}{\beta^{n}(\alpha-1)(\alpha-2) \ldots(\alpha-n)}, 1 \leq n<\alpha .
$$

The basket option is priced by approximating the sum of lognormal variables by an inverse gamma distribution. The first two moments are matched to get the two parameters of the inverse gamma density given by equation (7):

$$
\mu_{1}^{\prime}(v)=\mu_{1}^{\prime}\left(g_{R}\right)=\frac{1}{\beta(\alpha-1)} \text { and } \mu_{2}^{\prime}(v)=\mu_{2}^{\prime}\left(g_{R}\right)=\frac{1}{\beta^{2}(\alpha-1)(\alpha-2)}
$$

Using the inverse gamma density, the option price $P(t, T)$ $\int_{-\infty}^{+\infty} \max \left(x-K_{B}, 0\right) v(x) d x$ is approximated by:

$$
\begin{gathered}
V_{\text {gamma }}^{B}=P(t, T) \int_{K_{B}}^{+\infty}\left(x-K_{B}\right) g_{R}(x \mid \alpha, \beta) d x \\
=P(t, T) \int_{K_{B}}^{+\infty}\left(x-K_{B}\right) x^{-2} g\left(\frac{1}{x} \mid \alpha, \beta\right) d x \\
\left.=P(t, T) \int_{0}^{\frac{1}{K_{B}}}\left(\frac{1}{y}-K_{B}\right) g(y \mid \alpha, \beta) d y \text { (from the change of variable } y=\frac{1}{x}\right) \\
=P(t, T)\left(\frac{1}{\beta(\alpha-1)} \int_{0}^{\frac{1}{K_{B}}} g(y \mid \alpha-1, \beta) d y-K_{B} \int_{0}^{\frac{1}{K_{B}}} g(y \mid \alpha, \beta) d y\right) \\
=P(t, T)\left(\frac{1}{\beta(\alpha-1)}\left(G\left(\frac{1}{K_{B}} \mid \alpha-1, \beta\right)\right)-K_{B}\left(G\left(\frac{1}{K_{B}} \mid \alpha, \beta\right)\right)\right)
\end{gathered}
$$


where we use $\frac{1}{y} g(y \mid \alpha, \beta)=\frac{1}{\beta(\alpha-1)} g(y \mid \alpha-1, \beta)$. Equation (6) is therefore obtained.

\section{Edgeworth-lognormal Expansion}

This appendix shows how we obtain the pricing formula using an Edgeworth expansion around the lognormal distribution. Matching the first two moments, the lognormal density used is given by:

$$
a(x)=\frac{1}{\sqrt{2 \pi}} \frac{1}{\beta} \frac{1}{x} \exp \left(-\frac{1}{2}\left(\frac{1 n x-a}{\beta}\right)^{2}\right)
$$

where $\alpha$ and $\beta$ are defined by equations (8e) and (8f) respectively. Following Jarrow and Rudd (1982), the unknown basket density function can be approximated by:

$$
\begin{aligned}
v(x)= & a(x)+\frac{\kappa_{2}(v)-\kappa_{2}(a)}{2 !} \frac{d^{2} a(x)}{d x^{2}}-\frac{\kappa_{3}(v)-\kappa_{3}(a)}{3 !} \frac{d^{3} a(x)}{d x^{3}} \\
& +\frac{\left(\kappa_{4}(v)-\kappa_{4}(a)\right)+3\left(\kappa_{2}(v)-\kappa_{2}(a)\right)^{2}}{4 !} \frac{d^{4} a(x)}{d x^{4}}+\xi(x)(20)
\end{aligned}
$$

where $\xi(x)$ is an error term and $\kappa_{i}(h), i=\{1,2,3,4\}$ are the first four cumulants of the density function $h \in\{v, a\}$ defined by the system of equations (5). Jarrow and Rudd (1982) state that, in general, there is no bound on the error term resulting from an Edgeworth expansion. Consequently, the error does not necessarily decrease with the expansion's order. Given that the first two moments are the same for the true density and for the approximated density, equation (20) becomes:

$v(x)=a(x)-\frac{\kappa_{3}(v)-\kappa_{3}(a)}{3 !} \frac{d^{3} a(x)}{d x^{3}}+\frac{\kappa_{4}(v)-\kappa_{4}(a)}{4 !} \frac{d^{4} a(x)}{d x^{4}}+\xi(x)$.

Therefore the basket option price $P(t, T) \int_{-\infty}^{+\infty} \max \left(x-K_{B}, 0\right) v(x) d x$ can 
be approximated by:

$$
\begin{aligned}
V_{\log \text { normal }}^{B}= & P(t, T) \int_{K_{B}}^{+\infty}\left(x-K_{B}\right)\left(a(x)-\frac{\kappa_{3}(v)-\kappa_{3}(a)}{3 !} \frac{d^{3} a(x)}{d x^{3}}+\right. \\
& \left.\frac{\kappa_{4}(v)-\kappa_{4}(a)}{4 !} \frac{d^{4} a(x)}{d x^{4}}\right) d x .
\end{aligned}
$$

Using the fact that

$$
\int_{K_{B}}^{+\infty}\left(x-K_{B}\right) \frac{d^{j} a}{d x^{j}}(x) d x=\frac{d^{j-2} a}{d x_{B}^{j-2}}\left(K_{B}\right) \text { for } j \geq 2,
$$

we obtain

$$
V_{\log \text { normal }}^{B}=P(t, T)\left[\begin{array}{c}
\int_{K_{B}}^{+\infty}\left(x-K_{B}\right) a(x) d x-\frac{\kappa_{3}(v)-\kappa_{3}(a)}{3 !} \frac{d a}{d x}\left(K_{B}\right) \\
+\frac{\kappa_{4}(v)-\kappa_{4}(a)}{4 !} \frac{d^{2} a}{d x^{2}}\left(K_{B}\right)
\end{array}\right]
$$

Note that the integral in equation (22) is similar to Black and Scholes price.

\section{References}

Barraquand, J. 1995. Numerical Valuation of High Dimensional Multivariate European Securities. Management Science 41: 1882-1891.

Barton, D. E., and Dennis, K. E. R. 1952. The Conditions Under Which Gram-Charlier and Edgeworth Curves are Positive Definite and Unimodal. Biometrika 39: 425-427.

Bakshi, G., Cao, C., and Chen, Z. 1997. Empirical Performance of Alternative Option Pricing Models. Journal of Finance 52: 2003-2049.

Bakshi, G., Carr, P., and Wu, L. 2008. Stochastic Risk Premiums, Stochastic Skewness in Currency Options, and Stochastic Discount Factors in 
International Economies. Journal of Financial Economics 87: 132-156.

Bakshi, G., and Madan, D. A. 2006. Theory of Volatility Spreads. Management Science 52: 1945-1956.

Baxter, M., and Rennie, A. 1996. Financial Calculus: An Introduction to Derivative Pricing. Cambridge, New York.

Black, F., and Scholes, M. 1973. The Pricing of Options and Corporate Liabilities. Journal of Political Economy 81: 637-659.

Brigo, D., Mercurio, F., Rapisarda, F., and Scotti, R. 2004. Approximated Moment-Matching for Basket-Options Pricing. Quantitative Finance 4: 1-16.

Broadie, M., and Detemple, J. 1996. American Option Valuation: New Bounds, Approximations and a Comparison of Existing Methods. Review of Financial Studies 9: 1211-1250.

Curran, M. 1994. Valuing Asian and Portfolio Options by Conditioning on the Geometric Mean Price. Management Science 40: 1705-1711.

Dahl, L.O. 2000. Valuation of European Call Options on Multiple Underlying Assets by Using a Quasi-Monte Carlo Method. A Case with Baskets from Oslo Stock Exchange. In Proceedings AFIR 10: 239-248.

Dahl, L.O., and Benth, F.E. 2001. Valuation of Asian Basket Options with Quasi-Monte Carlo Techniques and Singular Value Decomposition. Pure Mathematics 5: 1-21.

Datey, J.Y., Gauthier, G., and Simonato, J.G. 2003. The Performance of Analytical Approximations for the Computation of Asian-Quanto-Basket Option Prices. Multinational Finance Journal 7: 55-82.

Deelstra, G., Liinev, J., and Vanmaele, M. 2004. Pricing of Arithmetic Basket Options by Conditioning. Insurance: Mathematics and Economics 34: 1-23.

Dionne, G., Gauthier, G., and Ouertani, N. 2008. Basket Options on Heterogeneous Underlying Assets. Working paper. Canada Research Chair in Risk Management. HEC Montréal.

Gentle, D. 1993. Basket Weaving. Risk 6: 51-52.

Gourieroux, C., and Sufana, R. 2004. Derivative Pricing with Multivariate Stochastic Volatility: Application to Credit Risk. Working paper. University of Toronto.

Harrison, J.M., and Pliska, S.R. 1981. Martingales and Stochastic Integrals in the Theory of Continuous Trading. Stochastic Processes and Their Applications 11: 261-271.

Heston, S.L. 1993. A Closed-form Solution for Options with Stochastic Volatility with Applications to Bond and Currency Options. Review of Financial Studies 6: 327-343.

Hill, I., Hill, R., and Holder, R. 1976. Fitting Johnson Curves by Moments. Applied Statistics 25: 180-192.

Hull, J., and White, A. 1987. Pricing of Options on Assets with Stochastic Volatilities. Journal of Finance 42: 281-300.

Huynh, C.B. 1994. Back to Baskets. Risk 5: 59-61. 
Jarrow, R., and Rudd, A. 1982. Approximate Option Valuation for Arbitrary Stochastic Processes. Journal of Financial Economics 10: 347-369.

Johnson, N.L. 1949. Systems of Frequency Curves Generated by Methods of Translation. Biometrika 36: 149-176.

Ju, N. 2002. Pricing Asian and Basket Options via Taylor Expansion. Journal of Computational Finance 5: 79-103.

Laurence, P., and Wang, T.H. 2004. What's a Basket Worth? Risk 17: 73-77.

Milevsky, M.A., and Posner, S.E. 1998a. Asian Options, the Sum of Lognormals and the Reciprocal Gamma Distribution. Journal of Financial and Quantitative Analysis 33: 409-422.

Milevsky, M.A., and Posner, S.E. 1998b. A Closed-Form Approximation for Valuing Basket Options. Journal of Derivatives 5: 54-61.

Milevsky, M.A., and Posner, S.E. 1999. Another Moment for the Average Option. Derivatives Quarterly 5: 47-53.

Pellizzari, P. 2001. Efficient Monte Carlo Pricing of European Options Using Mean Value Control Variates. Decisions in Economics and Finance 24: 107-126.

Posner, S.E., and Milevsky, M.A. 1999. Valuing Exotic Options by Approximating the SPD with Higher Moments. Journal of Financial Engineering 7: 109-125.

Rubinstein, M. 1994. Return to Oz. Risk 7: 67-71.

Sabanis, S. 2003. Stochastic Volatility and the Mean Reverting Process. Journal of Futures Markets 23: 33-48.

Schwartz, E.S. 1997. The Stochastic Behavior of Commodity Prices: Implications for Valuation and Hedging. Journal of Finance 52, 922-973.

Tahani, N. 2005. Exotic Options Pricing under Stochastic Volatility. Working paper. Canada Research Chair in Risk Management. HEC Montréal.

Vanmaele, M., Deelstra, G., and Liinev, J. 2004. Approximation of Stop-Loss Premiums Involving Sums of Lognormals by Conditioning on Two Variables. Insurance: Mathematics and Economics 35: 343-367.

Wan, H. 2002. Pricing American-Style Basket Options by Implied Binomial Tree. Working paper. Haas School of Business. University of California at Berkeley. 